\title{
Medication and Cognitive-Behavioral Therapy During Pregnancy
}

By Jack M. Gorman, MD

Pregnancy is often described as a time of emotional bliss. Morning sickness and other physical discomfort notwithstanding, pregnant women are traditionally said to "glow," euphoric about the impending addition to their lives. No doubt, this description was concocted by men. Although most women are perhaps happy to be pregnant, many are not. These include teenagers and women beset with overwhelming financial and social problems. For such women, having a baby may not represent life's ultimate fulfillment. Other women worry about the effect of a new baby. on their careers. Some obsess about whether they will make a good mother. For whatever reason, pregnancy can be an enormously stressful and, for a minority, a harrowing time of life.

If we add to the above psychosocial pressures the fact that, contrary to popular belief among many psychiatrists, a pregnant woman has about as much chance of developing a psychiatric illness as a nonpregnant woman, we can see why it is critical to take seriously the mental health problems of pregnancy. Depression, anxiety disorders, mania, psychosis, and eating disorders all occur during pregnancy and all pose challenges for the physician.

Traditionally, obstetricians and psychiatrists have not collaborated on research and clinical projects as extensively as, say, psychiatrists and neurologists. The first critical thing that needs to be done is to encourage obstetricians to recognize significant emotional and mental health problems in their pregnant patients and make the appropriate referrals. Sometimes, that can be to the nurse in the office; at other times, a psychiatrist is required. Once referred, there are many important things for a psychiatrist to consider. Generally, the biggest fear is that psychotropic medications will harm the fetus. This is not an inconsequential worry. Medications for mood, anxiety, and psychotic disorders have powerful effects on brain function. It may not be sufficient to show that a newborn exposed to psychotropic medication during fetal life has no physical deformities at birth. Rather, we must be concerned about possible adverse effects on the developing brain that may take years to manifest themselves.
Against these concerns about the potential teratogenicity of antidepressant, anti-anxiety, and antipsychotics are three important considerations:

First, animal studies and some clinical studies are beginning to show that a newborn can be adversely affected by the mother's emotional distress during pregnancy. Children born to depressed pregnant women may have low birth weights. In preclinical studies, exposing a pregnant animal to abnormal levels of stress increases not only her level of glucocorticoids but her newborn's as well. Studies have shown that animals exposed to such stressful fetal environments maintain hyperactive glucocorticoid responses to stress and are avoidant for the rest of their lives. Hence, not treating a psychiatric disorder during pregnancy can be teratogenic.

Next, psychotherapy is effective for many mental health problems during pregnancy. Studies have shown that interpersonal psychotherapy is useful for depressed pregnant women. I have treated pregnant women with panic disorder with cognitive behavioral psychotherapy successfully. Thus, the treatment of psychiatric illness during pregnancy does not necessarily mean the prescription of a psychotropic medication.

Finally, many psychotropics appear to be safe during pregnancy. One can never be entirely sure about this because physical abnormalities in newborns are relatively rare, making it difficult to know if a particular drug exposure elevates the risk. Also, as mentioned above, we have relatively little long-term data on children exposed to psychiatric medications during fetal life. Nonetheless, there are reassuring data for the safety in pregnancy of the typical antipsychotics, tricyclic and selective serotonin reuptake inhibitor antidepressants, and benzodiazepines. Only the anticonvulsants commonly used for bipolar disorder have been shown definitively to be teratogenic. If medication is necessary, it often can be given safely to pregnant women.

The articles in this month's CNS Spectrums go a long way toward clarifying many of the issues discussed above. We are grateful to Uriel Halbreich, MD, and Susan G. Kornstein, MD, for guest editing this issue and we hope to hear from you about your thoughts, cases, and suggestions. CNS

Dr. Gorman is the editor of this journal and Esther and Joseph Klingenstein Professor of Psychiatry and chair of the Department of Psychiatry at Mount Sinai School of Medicine in New York City. 